\title{
The Mini Astrophysical MeV Background Observatory (MAMBO) CubeSat Mission
}

\author{
Peter F. Bloser, W. Thomas Vestrand, Markus P. Hehlen, Lucas P. Parker, Darrel T. Beckman, Justin M. McGlown, \\ Lee Holguin, Kimberly K. Katko, James D. Sedillo, Anthony E. Nelson, Gregory K. Lee \\ Intelligence and Space Research Division, Los Alamos National Laboratory \\ P.O. Box 1663, Los Alamos, NM, 87545, USA; +1 (505) 664-0524 \\ pbloser@lanl.gov
}

\begin{abstract}
The origin of the cosmic diffuse gamma-ray (CDG) background in the $0.3-30 \mathrm{MeV}$ energy range is a mystery that has persisted for over 40 years. The Mini Astrophysical MeV Background Observatory (MAMBO) is a CubeSat mission concept motivated by the fact that, since the MeV CDG is relatively bright, only a small detector is required to make high-quality measurements of it. Indeed, the sensitivity of space-based gamma-ray instruments to the CDG is limited not by size, but by the locally generated instrumental background produced by interactions of energetic particles in spacecraft materials. Comparatively tiny CubeSat platforms provide a uniquely quiet environment relative to previous gamma-ray science missions. The MAMBO mission will provide the best measurements ever made of the MeV CDG spectrum and angular distribution, utilizing two key innovations: 1) low instrumental background on a 12U CubeSat platform; and 2) an innovative shielded spectrometer design that simultaneously measures signal and background. Enabling technologies include the use of compact silicon photomultipliers (SiPMs) for scintillator readout, and a tagged calibration source for real-time gain adjustment. We describe the MAMBO instrument, readout, commercial $12 \mathrm{U}$ bus systems, and mission concept in detail, including simulations and laboratory measurements demonstrating the key measurement concept.
\end{abstract}

\section{INTRODUCTION}

Gamma rays with energies in the range $\sim 0.3-10 \mathrm{MeV}$ provide unique and powerful signatures of nuclear processes throughout the Universe. The detection, analysis, and interpretation of these signatures has long been a core capability of Los Alamos National Laboratory. Studying MeV gamma rays of cosmic origin requires sensitive instrumentation placed above the Earth's atmosphere. However, the sensitivity of space-based gamma-ray sensors is severely limited by locally generated instrumental backgrounds, which are copiously produced by the interaction of energetic particles with spacecraft and instrument materials. Comparatively tiny CubeSat platforms, which have become widely available and increasingly capable in recent years, offer a uniquely "quiet" environment compared to traditional, large-scale gamma-ray science missions.

We present the Mini Astrophysical MeV Background Observatory (MAMBO) $)^{1,2}$, a new CubeSat mission under development at Los Alamos. Unlike most other CubeSat missions currently under development for highenergy astronomy (e.g., BurstCube ${ }^{3}$ or EIRSAT- $1^{4}$ ), MAMBO is not dedicated to the study of bright transient objects and will achieve high sensitivity above energies of $1 \mathrm{MeV}$. MAMBO will take advantage of the low instrumental background afforded by a commercial $12 \mathrm{U}$ CubeSat bus to record the best measurement ever made of the cosmic diffuse gamma-ray (CDG) background in the $0.3-10 \mathrm{MeV}$ energy range. This mission represents a new way of doing $\mathrm{MeV}$ astronomy, taking advantage of a small mission's unique advantages for a specific scientific goal, while joining a new wave of rapid, relatively inexpensive space science missions enabled by CubeSats.

\section{SCIENTIFIC MOTIVATION}

Diffuse cosmic background radiation (the combined emission of sources too distant to be resolved into individual objects) provides a powerful constraint on the evolution of stars and galaxies over the history of the Universe. This cosmic emission is generally well understood - except for gamma rays from $\sim 0.3-100$ $\mathrm{MeV}$. The available data (Figure 1) indicate abrupt changes in the slope of the CDG energy spectrum at $\sim 3$ - $4 \mathrm{MeV}$, and again at $\sim 50-60 \mathrm{MeV}$, hinting that perhaps multiple source populations are involved. Certain massive accreting black holes called "blazars" are known to emit $\mathrm{MeV}$ gamma rays via particle acceleration and must play a role ${ }^{5}$. $\mathrm{MeV}$ gamma rays are also produced by nuclear processes in the stellar explosions known as Type Ia supernovae ( $\mathrm{SNe}$ Ia), which surely also contribute ${ }^{6}$. 


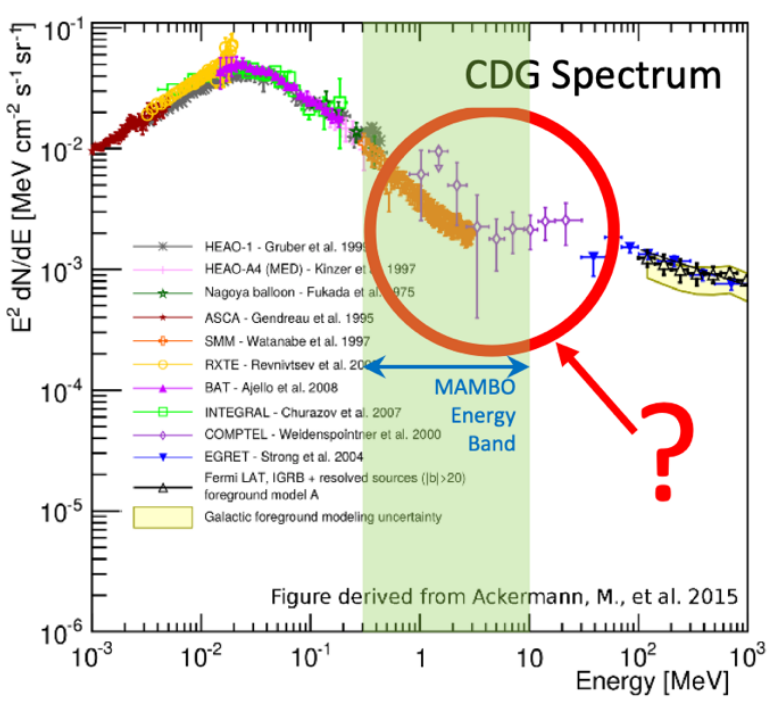

Figure 1: Existing measurements ${ }^{7}$ of the CDG in the $\mathrm{MeV}$ range have large uncertainties

The relative contributions of accretion processes and nuclear processes depend on the complex cosmological histories of galaxy evolution and star formation, respectively, as well as the detailed high-energy physics that produces the observed gamma rays. Resolving this decades-old puzzle will thus provide constraints on the evolution of the basic building blocks of the Universe ${ }^{8}$. The contributions of cosmic nuclear sources are of particular interest to Los Alamos, which is putting significant effort into advancing the accuracy of nuclear yield calculations, and the resulting gamma-ray signatures, for astrophysical explosions; the CDG provides a means of constraining these models that does not rely on observing a rare, transient event like a nearby supernova. The answers lie in the intensity and spectral shape of the CDG (revealing physical processes), as well as in the angular fluctuations in intensity across the sky (revealing source spatial distribution). At the moment, however, sufficient data do not exist.

The MeV CDG was first measured in the 1970s by simple spectrometers on the Apollo 15 \& 16 Lunar missions ${ }^{9}$. The best measurements to date were made by the Compton Telescope (COMPTEL) on NASA's Compton Gamma-Ray Observatory (CGRO) ${ }^{10}$ and the Solar Maximum Mission (SMM) Gamma-Ray Spectrometer ${ }^{11}$. Both analyses relied on complex procedures for estimating and subtracting time-variable instrumental backgrounds, both prompt and activation. Nearly order-of-magnitude systematic error bars are evident in the COMPTEL data (purple points in Figure 1), especially below $4 \mathrm{MeV}$ where activation is most prominent. The SMM data (orange points in Figure 1) show better statistical precision up to $3 \mathrm{MeV}$, but because this instrument continually monitored for solar flares, it could only observe a limited region of the sky centered on the Sun, preventing searches for anisotropy. What is required is a full-sky map of the $\mathrm{MeV}$ diffuse spectrum and anisotropy collected with a lowbackground instrument.

\section{THE MAMBO APPROACH}

\section{Instrumental Background in Space-Based Gamma- Ray Instruments}

The primary obstacle to solving this mystery is the poor quality and limited sky coverage of available observations in the $\mathrm{MeV}$ range (Figure 1). Because most discrete astrophysical gamma-ray sources are faint, the traditional approach to $\mathrm{MeV}$ astronomy relies on placing instruments with large collecting areas into space. This results in massive $\left(10^{3}-10^{4} \mathrm{~kg}\right)$ spacecraft busses and instrument structures; NASA's CGRO had a mass of $17,000 \mathrm{~kg}$. Energetic particles in space, including galactic cosmic rays and solar energetic particles, interact with these massive structures (aluminum in particular) to produce an intense background "fog" of secondary gamma rays and neutrons. This instrumental background can be prompt or delayed (i.e., resulting from activation), and it severely impacts the sensitivity of space-based instruments. It is especially pernicious for measurements of the $\mathrm{CDG}$, since both signal and background are diffuse and cannot be distinguished using techniques such as imaging or spectroscopy. Unfortunately, future NASA mission concepts under development are also massive and would be subject to complex backgrounds.

\section{The Advantages of CubeSats}

A low-mass spacecraft produces less instrumental background than a high-mass one. The exact scaling is dependent in a complex way on the mass composition and distribution, but a conservative estimate can be made by considering uniform cylinders of aluminum, representing the spacecraft bus, with the detector located at the center of one end (Figure 2a). Assuming a uniform emissivity due to prompt interactions and activation, the background is then proportional to the integral over the volume of $1 / d^{2}$, where $d$ is the distance to the detector. Performing this calculation for aluminum cylinders with masses from $10 \mathrm{~kg}$ to $10,000 \mathrm{~kg}$, the integrated "background" scales as (mass) $)^{1 / 3}$. In order to significantly reduce the instrumental background, therefore, the spacecraft mass must be dramatically reduced. CubeSats offer the only way to accomplish this.

MAMBO will utilize a commercial $12 \mathrm{U}$ CubeSat bus with dimensions $30 \times 20 \times 20 \mathrm{~cm}^{3}$ and a maximum mass of $24 \mathrm{~kg}$, two to three orders of magnitude less than spacecraft historically used for astrophysics missions 
(a)

\section{Uniform Al Cylinders:}

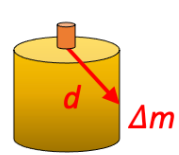

Background $\sim \int_{V}\left(1 / d^{2}\right) d V \sim(\text { mass })^{1 / 3}$

(b)

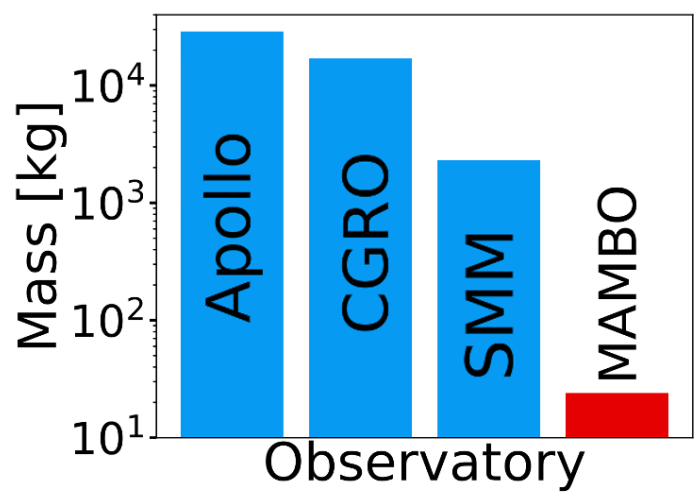

Figure 2: (a) Instrumental background scales approximately as (mass) $)^{1 / 3}$ (b) As a 12U CubeSat mission, MAMBO will have 2-3 orders of magnitude lower mass than previous missions which observed the CDG

(Figure 2b). Such a low-mass spacecraft will produce approximately an order of magnitude less instrumental background than was seen by COMPTEL. This approach is practical for the science goals described above due to the fact that the $\mathrm{MeV} \mathrm{CDG}$ is relatively bright, and thus does not require a large detector for a high-quality measurement.

\section{THE MAMBO PAYLOAD}

\section{The MAMBO Instrument}

The MAMBO instrument is illustrated in Figures 3 and 4. MAMBO achieves high efficiency and exceptional background rejection using an innovative shielding configuration. The MAMBO spectrometer employs two identical, closely-spaced Bismuth Germanate (BGO) scintillator detectors enclosed within an active anticoincidence cup, also of BGO. The cup is capped at the rear with $\mathrm{BGO}$, and at the entrance with a thin anti- coincidence plastic scintillator to reject charged particles while allowing gamma rays to enter. Since the two BGO detectors are identical with a common gain monitored by a calibration source, we can use the back detector as a real-time monitor of the internal instrumental background. The instrument is able to fit within the size, weight, and power (SWaP) confines of a 12U CubeSat through the use of silicon photomultiplier (SiPM) readouts.

The front scintillator, denoted the Primary Detector, is a $3^{\prime \prime}$ (diameter) $\times 2.5^{\prime \prime}$ (length) cylinder of BGO and is exposed to the CDG through the entrance aperture (Fig. 3). The rear, identical, BGO is designated the Background Monitor and is shielded from the CDG by the Primary Detector. Both detectors are exposed to the same instrumental background from the sides. Subtraction of "Monitor" spectra from "Primary" spectra thus removes the instrumental background even during

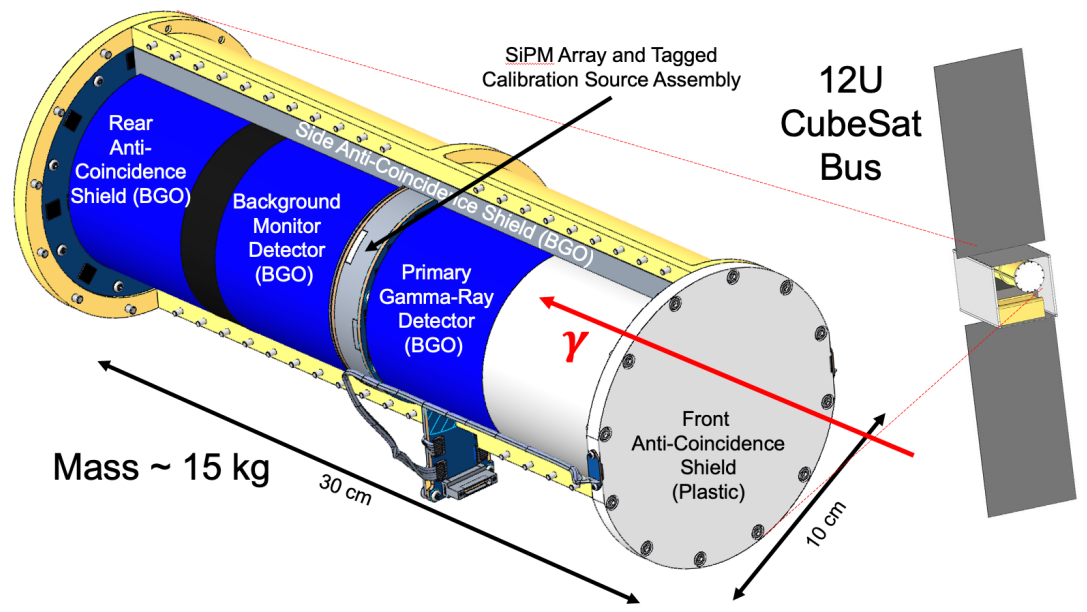

Figure 3: The MAMBO instrument achieves high efficiency in a compact configuration suited for deployment on a $12 \mathrm{U}$ CubeSat bus 
fast variations, a fundamentally new approach to the traditional practice of calculating or simulating the background. Cosmic radiation incident from the sides is subtracted as well, suppressing the response to off-axis sources. Sources of radiation outside the field of view are also suppressed by the surrounding Side and Rear BGO anti-coincidence shields.

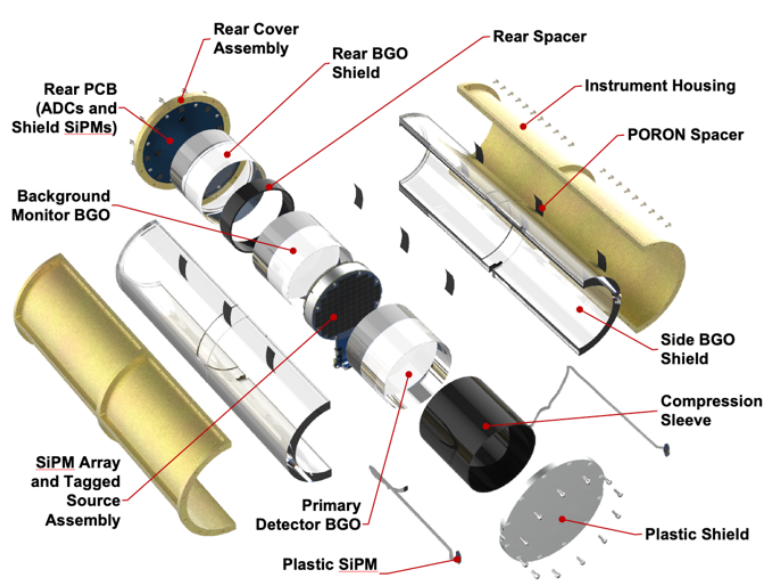

Figure 4: Exploded view of the MAMBO instrument

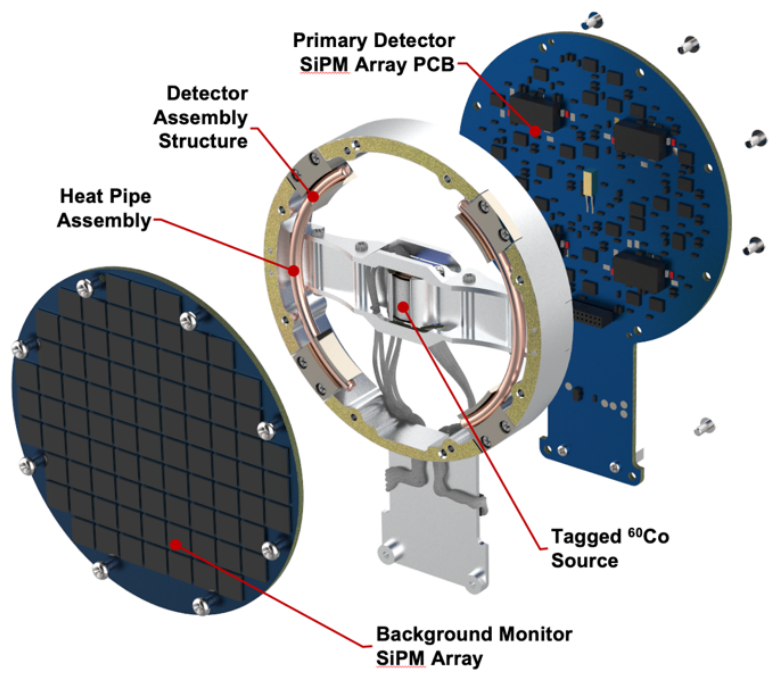

Figure 5: MAMBO SiPM array and tagged calibration source assembly

To enable CubeSat deployment, the scintillator light output is measured by SiPMs (Figures 4 and 5). The Primary Detector and Background Monitor are read by identical, custom arrays of SiPMs which are designed for maximum compactness and sum the individual array elements into a single output signal for each scintillator. The MICROFJ-60035-TSV device, a $6 \mathrm{~mm} \times 6 \mathrm{~mm}$ "Jseries" SiPM manufactured by SensL/ON Semiconductor, will be used to construct these arrays. The anti-coincidence shields are read out by individual
SiPMs mounted on small printed circuit boards (PCBs) in the case or the plastic shield, or by summed SiPMs on a "Rear PCB" for the Side and Rear BGO Shields; these signals "veto" the processing of coincident Primary Detector or Background Monitor events. As SiPM gain is dependent on temperature, the readout system will monitor thermistors and coarsely adjust the bias voltage of the shield SiPMs in order to maintain an approximately constant gain.

It is critical that the gains of the Primary Detector and Background Monitor be kept precisely matched in order for the MAMBO background subtraction technique to work. This is accomplished using a "tagged" calibration source (Figure 5): a small button of plastic scintillator infused with ${ }^{60} \mathrm{Co}$, with its own dedicated SiPM readout, produces a flash of light due to the $\beta$-particle emitted in coincidence with $1.17 \mathrm{MeV}$ and $1.33 \mathrm{MeV}$ gamma rays. This identifies calibration events in each of the two main detectors; these events are analyzed by an algorithm running on a Field Programmable Gate Array (FPGA) to determine necessary bias voltage adjustments to maintain matched and constant gains. This technique was successfully used in space on $\mathrm{SMM}^{12}$.

\section{Payload Readout}

The MAMBO payload readout and control system is currently being designed in detail. A conceptual block diagram is shown in Figure 6.

The "Rear PCB" contains the analog-to-digital and coincidence electronics for the MAMBO instrument. The summed signal pulses from the Primary Detector and Background Monitor SiPM arrays are digitized by $50 \mathrm{MHz}$ waveform-digitizing analog-to-digital converters (ADCs). This sampling rate is sufficient for the relatively slow ( $\sim 300 \mathrm{~ns})$ light decay time of the BGO scintillators. The Rear PCB also collects signals from the readout SiPMs on the front plastic anti-coincidence shield, and houses the readout SiPMs for the Side and Read BGO shields. These signals are compared to controllable threshold voltages set by digital-to-analog converters (DACs) and then trigger coincidence logic to set anti-coincidence flags when shield signals occur within a (programmable) time window around a pulse from the Primary Detector and/or Background Monitor. Similarly, signals from the tagged ${ }^{60} \mathrm{Co}$ source generate a flag to identify calibration events.

Two additional electronics boards are housed in a SpaceVPX 3U Card Box (note that the "U" designation for electronic board form factor is not related to the CubeSat "U" categories), connected by a backplane. The low-voltage power supply (LVPS) and SiPM Bias Voltage Board converts the available voltages from the commercial $12 \mathrm{U}$ spacecraft bus into the required 


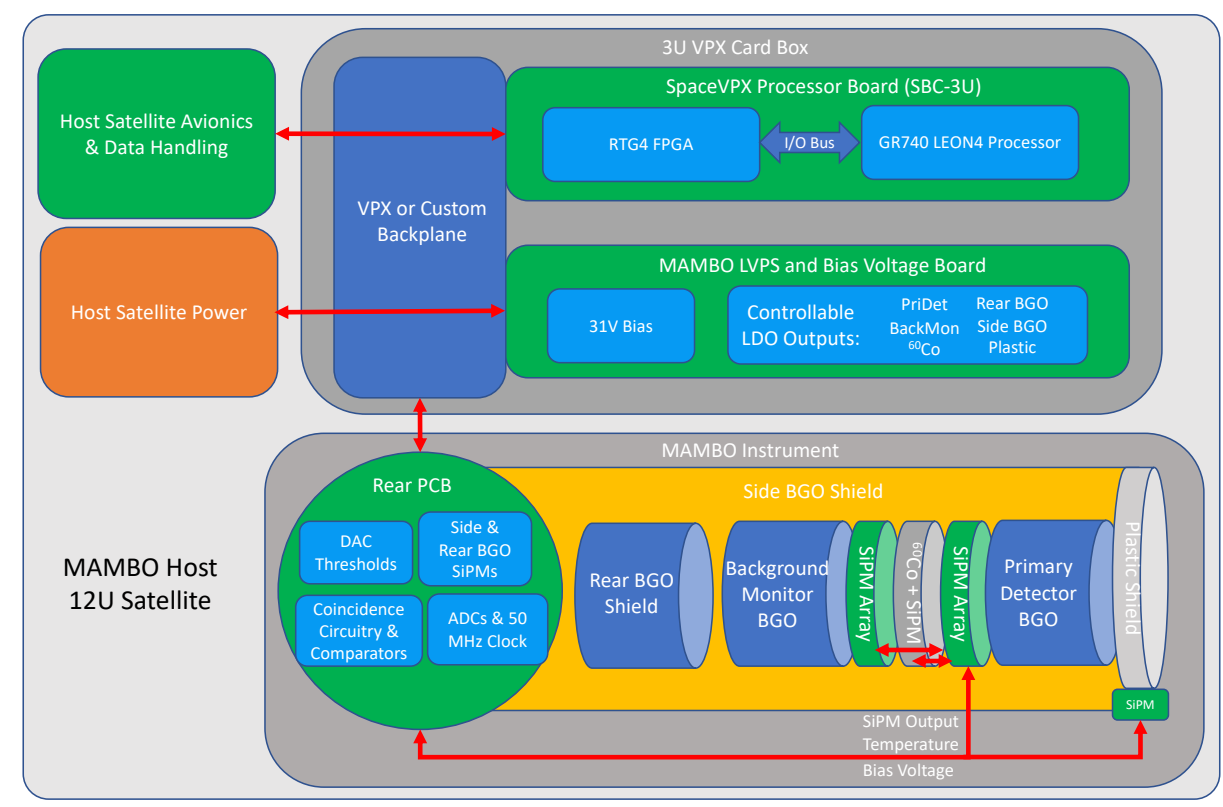

Figure 6: Block diagram of the MAMBO readout and control system

electronics levels, and generates an adjustable (25 V - 31 V) bias voltage for each of the SiPM readouts in the instrument. The bias levels for the shield and tagged ${ }^{60} \mathrm{Co}$ source SiPMs will be adjusted according to the temperature, as reported by sensors co-located with the SiPMs, via look-up tables. As described above, the bias for the Primary Detector and Background Monitor SiPM arrays will be calculated via an analysis of the tagged ${ }^{60}$ Co event pulse heights.

The SpaceVPX Processor Board controls the payload functions and communicates with the commercial $12 \mathrm{U}$ bus avionics. This board will be a smaller, 3U-sized version of a radiation-hardened $6 \mathrm{U}$ processor currently under development at Los Alamos ${ }^{13}$. The system controller is based on the space grade GR740 quad-core LEON4 processor ASIC with a MicroChip RTG4 FPGA, which will communicate with the ADCs and coincidence logic on the instrument Rear PCB and will implement the real-time SiPM bias adjustment based on analysis of the tagged ${ }^{60} \mathrm{Co}$ events. The flight software will assemble event-mode data for the Primary Detector and Background Monitor, including pulse heights, time tags, and flags indicating coincidence with each other and/or the tagged ${ }^{60} \mathrm{Co}$ source and anti-coincidence shields. The Processor will also collect rates from each detector element and state-of-health data from the payload (currents, voltages, and temperatures), as well as communicate with the host bus avionics to receive commands and send out data for telemetry to the ground.

One possible mechanical accommodation of the MAMBO instrument and SpaceVPX Card Box in a generic $12 \mathrm{U}$ CubeSat bus is shown in Figure 7. There is ample volume available, and current best estimates of the mass and power are within the constraints imposed by the commercial vendor we have selected. We will work with our commercial partner to make the satellite mass distribution as symmetric as possible so that the instrumental background recorded by the Primary Detector and Background Monitor are nearly identical.

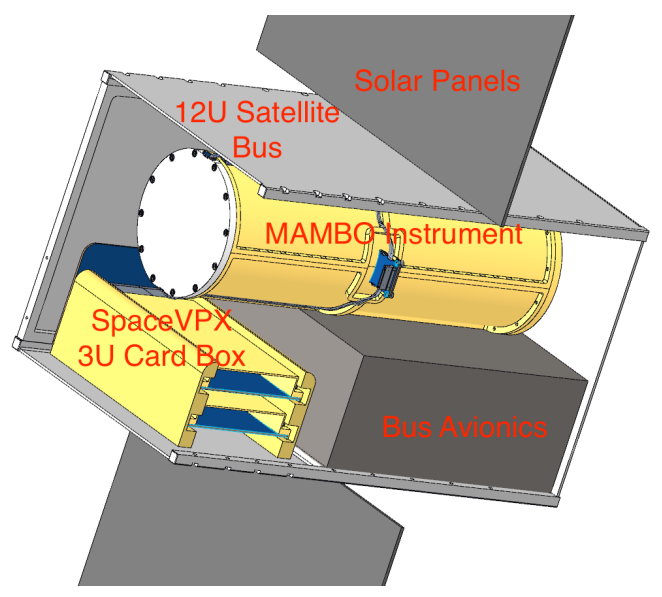

Figure 7: Mechanical accommodation of the MAMBO payload in a generic $12 \mathrm{U}$ CubeSat bus VALIDATION STUDIES

We have performed laboratory measurements and Monte Carlo computer simulations to validate the MAMBO measurement principle and to predict the instrument response and expected background counting rates in orbit. 


\section{Laboratory Measurements}

As presented previously ${ }^{2}$, two 3-inch cylindrical BGO scintillators were placed adjacent to each other in the MAMBO configuration and read out with commercial SiPM arrays (Figure 8 inset). Spectra were recorded from each as a ${ }^{60} \mathrm{Co}$ source of 1.17 and $1.33 \mathrm{MeV}$ gamma rays was placed at different "zenith angles" relative to the axis or symmetry. After subtracting the Monitor (B) detector counts from the Primary (A), the combined efficiency falls off with zenith angle (Figure 8), with a half-response angle of $\sim 30^{\circ}(\sim 0.85 \mathrm{sr})$.

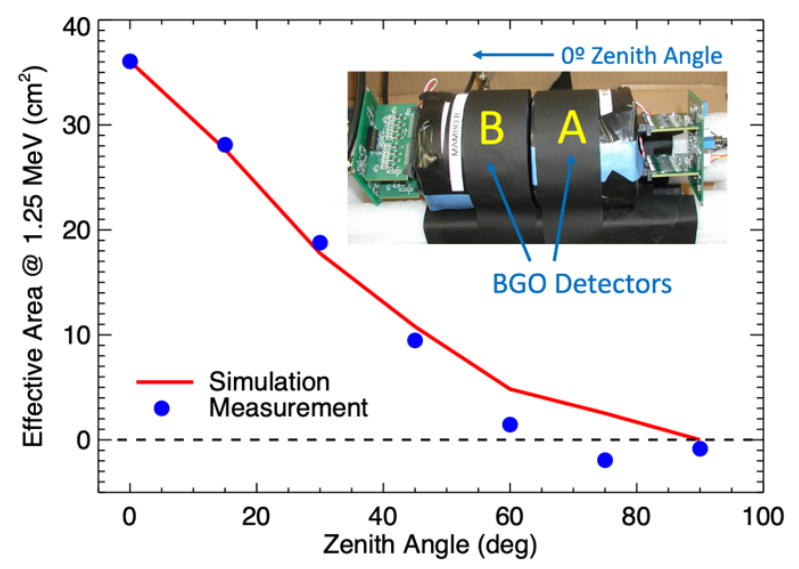

Figure 8: Laboratory \& simulation tests of MAMBO background subtraction approach

\section{Simulations}

The laboratory test results are in good agreement with simulations performed using the MEGAlib software package $^{14}$. The laboratory setup was replicated in software for incident $1.25 \mathrm{MeV}$ photons (average of the two ${ }^{60} \mathrm{Co}$ energies), and after subtracting the " $\mathrm{B}$ " detector counts from the "A" detector the fall-off in effective area agrees with the data for zenith angles up to $50^{\circ}$ (Figure 8). For angles above $60^{\circ}$ slight disagreement is seen, likely due to imperfect alignment and slightly mismatched gains.

We next simulated the response of the MAMBO instrument to incident gamma rays of several energies as a function of incidence angle. The MEGAlib "mass model" and the effective area (after subtraction of the Background Monitor counts from the Primary Detector counts) are shown in Figure 9. The MAMBO instrument attains good efficiency for energies up to $10 \mathrm{MeV}$, with an effective area of $\sim 32 \mathrm{~cm}^{2}$ at $1 \mathrm{MeV}$ and a field of view of $\sim 40^{\circ}$ (FWHM). This effective area is roughly the same as that of the much larger COMPTEL telescope, after all imaging and background rejection data selections were applied. MAMBO will therefore be able to measure the CDG spectrum in the $\mathrm{MeV}$ energy band at multiple locations in the sky.
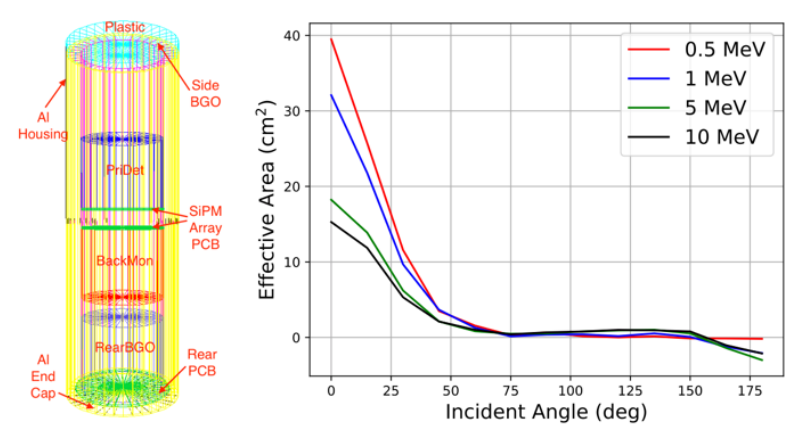

Figure 9: Simulation "mass model" of the MAMBO instrument, with simulated effective area vs. incidence angle for several energies

Finally, we created a mass model of the MAMBO payload in a generic $12 \mathrm{U}$ CubeSat bus and simulated the response to the expected background radiation fields in a low-Earth orbit (LEO) for several inclinations. We used input spectra collected and formatted for MEGAlib $^{15}$, and included the effects of activation by trapped protons in the Earth's radiation belts. For the case of a $28^{\circ}$ inclination orbit at an altitude of $500 \mathrm{~km}$, with the instrument pointed $30^{\circ}$ off of zenith, the predicted counting rate spectra due to different radiation inputs are shown in Figures 10 and 11. In Figure 10, the spectra recorded by the Primary Detector only are shown, using all other scintillators are active shielding only. The recorded counts are dominated by activation from trapped hadrons and albedo photons from the Earth's atmosphere.

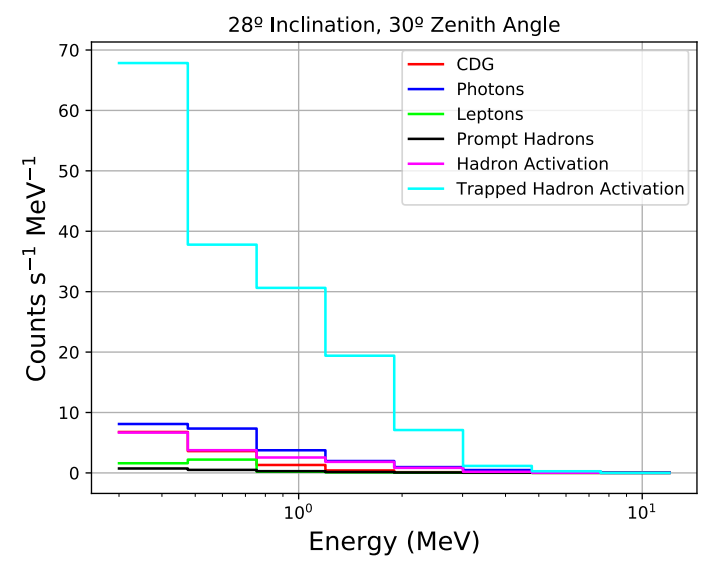

Figure 10: Simulated background spectra in LEO in the Primary Detector, without subtracting counts from the Background Monitor

In Figure 11, the MAMBO background subtraction technique is applied. The CDG counts (red curve) from the instrument field of view are far more prominent after background subtraction, illustrating the value of the MAMBO approach in increasing sensitivity. 


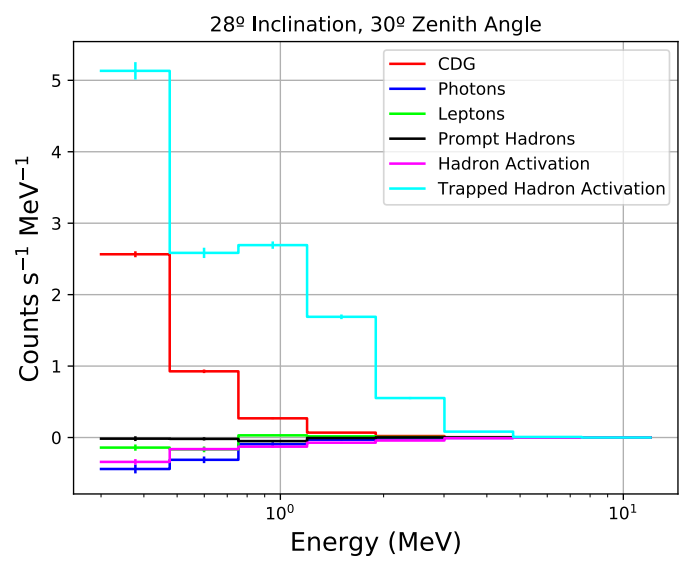

Figure 11: Simulated background spectra in LEO after subtracting the Background Monitor counts from the Primary Detector

\section{THE MAMBO MISSION}

The ideal science orbit for MAMBO would be an equatorial LEO with an altitude of $\sim 400-500 \mathrm{~km}$ and an inclination of $\sim 0^{\circ}$, as this would minimize exposure to the Earth's radiation belts. For practical reasons related to the project schedule, however, we will accept launches to orbits with inclinations up to that of the International Space Station $\left(\sim 52^{\circ}\right)$, as the MAMBO background subtraction technique should permit sensitive observations of the MeV CDG in this orbit as well. Because solar activity is expected to increase in the coming years, we also baseline an altitude of $500 \mathrm{~km}$ so as to guarantee an orbital lifetime of at least two years.

After an initial orbital checkout period, including pointed observations of well-studied sources such as the Crab Nebula to verify the expected instrument response, MAMBO will map the sky form $0.3-10 \mathrm{MeV}$ via a series of approximately 25 pointed observations lasting $\sim 10^{6}$ seconds each. Time-variable backgrounds will be extrapolated to zero to reveal the residual constant signal from the CDG, following and building on the analyses of COMPTEL $^{10}$ and $\mathrm{SMM}^{11}$. The instrument field of view is $\sim 1$ steradian; the overlap between the 25 pointings will allow us to mitigate contamination from bright point sources and the Galactic Plane. We note that relatively few sources exist at high Galactic latitudes that are bright in this energy range. The required pointing accuracy is modest, $\sim 1^{\circ}$. The background simulations described above predict that the total counting rates in the two main BGO detectors should never exceed $500 \mathrm{cts} \mathrm{s}^{-1}$ outside of the radiation belts, and from this the estimated data volume is $\lesssim 600 \mathrm{MB}$ per day. MAMBO will use a commercial or government ground station network for telemetry and commanding; detailed requirements are under development and we will select a ground station partner in the coming months.

The MAMBO project timeline is shown in Figure 12. A six-month Feasibility Study was completed to demonstrate key enabling technologies and verify the expected science return using simulations. After passing a Feasibility Review in March 2021, the project is currently in a one-year period of instrument development and $12 \mathrm{U}$ bus procurement, to be followed by one year of integration and test. Following launch in 2023 a oneyear baseline mission will take place, with the possibility of extended science operations if additional external (e.g. NASA) funding can be secured. The mission duration required to achieve minimum science goals (measurement of the CDG spectrum at the Galactic north and south poles) is estimated to be six months. The MAMBO mission timeline will continue to be refined in consultation with the selected commercial CubeSat vendor. Launch services are being secured via the Department of Defense's Space Test Program.

\section{SUMMARY}

The MAMBO 12U CubeSat mission is under development at Los Alamos and will provide the best measurement ever made of the $\mathrm{CDG}$ in the $\mathrm{MeV}$ energy band. MAMBO will also demonstrate the utility of commercial 12U satellite buses and ground station networks for rapid, low-cost space missions.

\section{Acknowledgments}

Research presented in this article was supported by the Laboratory Directed Research and Development program of Los Alamos National Laboratory under project number 20210047DR.

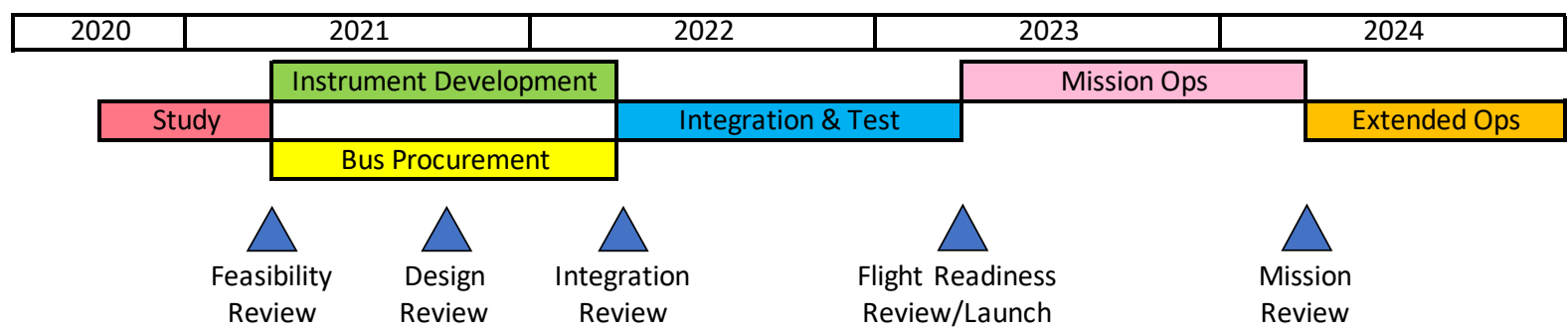

Figure 12: The MAMBO project timeline 


\section{References}

1. Vestrand, W. T., Bloser, P. F., Hoover, A. S., Parker, L. P., and Wren, J., "The Mini Astrophysical $\mathrm{MeV}$ Background Observatory (MAMBO)," 36th International Cosmic Ray Conference (ICRC2019), Madison, WI, JulyAugust 2019.

2. Bloser, P. F., Vestrand, W. T., Hehlen, M. P., Parker, L. P., and Hoover, A. S., "The Mini Astrophysical MeV Background Observatory (MAMBO) CubeSat mission," Proc. SPIE 11444, Space Telescopes and Instrumentation 2020: Ultraviolet to Gamma Ray, 114441Z (13 December 2020).

3. Jeremy S. Perkins, Isabella Brewer, Michael S. Briggs, Alessandro Bruno, Eric Burns, Regina Caputo, Brad Cenko, Antonino Cucchiara, Georgia De Nolfo, Jeff Dumonthier, Sean Griffin, Lorraine Hanlon, Dieter H. Hartmann, Boyan Hristov, Michelle Hui, Alyson Joens, Carolyn Kierans, Marc Kippen, Dan Kocevski, John Krizmanic, Sibasish Laha, Amy Lien, Israel Martinez- Castellanos, Sheila McBreen, Julie E. McEnery, J. G. Mitchell, Lee Mitchell, David Morris, David Murphy, Judith L. Racusin, Oliver Roberts, Peter Shawhan, Jacob Smith, George Suarez, Teresa Tatoli, Alexey Uliyanov, Carlos Vazquez, Sarah Walsh, Colleen Wilson-Hodge, "BurstCube: a CubeSat for gravitational wave counterparts," Proc. SPIE 11444, Space Telescopes and Instrumentation 2020: Ultraviolet to Gamma Ray, 114441X (13 December 2020).

4. Sarah Walsh, David Murphy, Maeve Doyle, Joseph Thompson, Rachel Dunwoody, Masoud Emam, Jessica Erkal, Joe Flanagan, Gianluca Fontanesi, Andrew Gloster, Joe Mangan, Conor O'Toole, Favour Okosun, Rakhi Rajagopalan Nair, Jack Reilly, Lána Salmon, Daire Sherwin, Paul Cahill, Daithí de Faoite, Umair Javaid, Lorraine Hanlon, David McKeown, William O'Connor, Kenneth Stanton, Alexei Ulyanov, Ronan Wall and Sheila McBreen, "Assembly, Integration and Verification Activities for a $2 \mathrm{U}$ CubeSat, EIRASAT-1" 3rd Symposium on Space Educational Activities, Leicester, UK (September 16-18, 2019).

5. M. Ajello, L. Costamante, R. M. Sambruna, N. Gehrels, J. Chiang, A. Rau, A. Escala, J. Greiner, J. Tueller, and J. V. Wall, "The Evolution of Swift/BAT Blazars and the Origin of the $\mathrm{MeV}$ Background," Astrophysical Journal, 699, 603, 2009.
6. Ruiz-Lapuente, P., The, L.-S., Hartmann, D. H., Ajello, M., Canal, R., Röpke, F. K., Ohlmann, S. T., and Hillebandt, W., "The Origin of the Cosmic Gamma-Ray Background in the MeV Range," Astrophysical Journal, 820, 142, 2016.

7. Ackermann, M., et al., "The Spectrum of Isotropic Diffuse Gamma-Ray Emission Between $100 \mathrm{MeV}$ and $820 \mathrm{GeV}$," Astrophysical Journal, 799, 86, 2015.

8. Marco Ajello, Yoshiyuki Inoue, Peter Bloser, W. T. Vestrand, Dieter Hartmann, Justin Finke, Zorawar Wadiasingh, Chanda Prescod-Weinstein, Uwe Oberlack, Francesco Longo, Chris Shrader, Volker Beckmann, Ivan Agudo, Maria Petropoulou, Lih-Sin The, A. Strong, Eugenio Bottacini, John W. Hewitt, Alexander Moiseev, Sean Griffin, Dario Gasparrini, and John Tomsick, "The MeV Background," Astro2020: Decadal Survey on Astronomy and Astrophysics, science white papers, no. 290; Bull. American Astronomical Society, Vol. 51, Issue 3, id. 290 (2019).

9. Trombka, J. I., Dyer, C. S., Evans, L. G., Bielefeld, M. J., Seltzer, S. M., and Metzger, A. E., "Reanalysis of the Apollo cosmic gamma-ray spectrum in the 0.3 to $10 \mathrm{MeV}$ energy region," Astrophysical Journal, 212, 925, 1977.

10. Weidenspointner, G., Varendorff, M., Kappadath, S. C., Bennett, K., Bloemen, H., Diehl, R., Hermsen, W., Lichti, G. G., Ryan, J., and Schönfelder, V., "The cosmic diffuse gamma-ray background measured with COMPTEL," AIP Conf. Proc. 510, The Fifth Compton Symposium, ed. M. L. McConnell \& J. M. Ryan (Melville, NY: AIP), 581 (2000).

11. Watanabe, K., Leising, M. D., Share, G. H., and Kinzer, R. L., "The MeV cosmic gamma-ray background measured with SMM," AIP Conference Proceedings 510, The Fifth Compton Symposium, ed. M. L. McConnell \& J. M. Ryan (Melville, NY: AIP), 471 (2000).

12. Forrest, D. J., Chupp, E. L., Ryan, J. M., Cherry, M. L., Gleske, I. U, Reppin, C., Pinkau, K., Rieger, E., Kanbach, G., Kinzer, R. L., Share, G., Johnson, W. N., and Kurfess, J. D., "The Gamma Ray Spectrometer for the Solar Maximum Mission", Solar Physics, 65, 15, 1980.

13. Merl, R., Cox, E., Dutch, R., Graham, P., Larsen, S., Michel, J., Milby, D., Morgan, K., and Tripp, K., "LEON4 Based Radiation-Hardened SpaceVPX System Controller," 2020 IEEE Aerospace Conference, Big Sky, Montana (March 2020). 
14. Zoglauer, A., Andritschke, R., and Schopper, F., "MEGAlib: The Medium Energy Gamma-ray Astronomy Library," New Astronomy Reviews, 50, 629, 2006.

15. Cumani, P., Hernanz, M., Kiener, J., Tatischeff, V., and Zoglauer, A., "Background for a gammaray satellite on a low-Earth orbit," Experimental Astronomy, 47, 273, 2019. 